\title{
Exchange proteins directly activated by cyclic adenosine monophosphate inhibitor reverses mechanical allodynia via the modification of astrocytes activity in the spinal cord
}

\author{
Qing She", Junjie Chen", Chaochao Zhong", Saisai Huang, Cui'e Lu, Yibin Qin \\ Department of Anesthesiology, Affiliated Hospital of Nantong University, Nantong, China \\ Contributions: (I) Conception and design: C Lu; (II) Administrative support: Y Qin; (III) Provision of study materials or patients: Q She; (IV) \\ Collection and assembly of data: Q She, J Chen, C Zhong; (V) Data analysis and interpretation: S Huang, C Lu, Y Qin; (VI) Manuscript writing: All \\ authors; (VII) Final approval of manuscript: All authors. \\ "These authors contributed equally to this work. \\ Correspondence to: Cui'e Lu; Yibin Qin. Department of Anesthesiology, Affiliated Hospital of Nantong University, No. 20 Xisi Road, Nantong, China. \\ Email: lucuie608@163.com; jsntqyb@163.com.
}

\begin{abstract}
Background: Gliosis and inflammation are pivotal in the development of acute and chronic pain. Here, we demonstrated a previously unidentified molecular mechanism in which the activation of exchange proteins directly activated by cyclic adenosine monophosphate (Epac) 1 accelerated the activation of astrocytes in the spinal cord, thereby promoting chronic postsurgical pain (CPSP).

Methods: We established a rat model of CPSP induced by skin/muscle incision and retraction (SMIR). Pain behaviors were assessed using mechanical withdrawal threshold (MWT) at different times. The lumbosacral enlargement of the spinal cord was isolated to detect the expression of Epac1 and the activity of astrocytes. They were assessed using western blot and immunofluorescence staining.

Results: SMIR induced persistent mechanical hyperalgesia after surgery. This hyperalgesia response was prolonged to more than $21 \mathrm{~d}$ after surgery. The time course of spinal Epac1 upregulation was correlated with SMIR-induced pain behaviors. Meanwhile, Epac1 immunoreactivity was colocalized primarily with astrocytes but not with microglial cells or neurons on $7 \mathrm{~d}$ after surgery. Intrathecal injection of Epac1 inhibitor CE3F4 significantly suppressed SMIR-induced mechanical allodynia and activation of astrocytes in the spinal cord. This analgesic effect of single-dose administration of CE3F4 lasted up to $6 \mathrm{~h}$ and wore off at $12 \mathrm{~h}$ after injection.
\end{abstract}

Conclusions: Spinal Epac1-mediated activation of astrocytes may facilitate CPSP. Inhibition of Epac1 may effectively prevent CPSP.

Keywords: Epac1; spinal cord; astrocytes; skin/muscle incision and retraction (SMIR); chronic postsurgical pain (CPSP)

Submitted Aug 22, 2021. Accepted for publication Nov 09, 2021.

doi: 10.21037/atm-21-5384

View this article at: https://dx.doi.org/10.21037/atm-21-5384

\section{Introduction}

Chronic postsurgical pain (CPSP) is a potential adverse outcome after surgery with an estimated incidence up to $50 \%$ of individuals after surgeries (1). This chronic pain is accompanied by depression, disability, and reduced quality of life. The development of CPSP is multifactorial (2), and it is still a basic science and clinical challenge (3). Peripheral sensitization and central sensitization induced by persistent changes of maladaptive neuroplasticity are involved in the process of CPSP $(4,5)$. However, the underlying cellular and molecular mechanisms are still unclear, and this is a major limitation for identification of novel and adequate 
treatments.

Exchange proteins directly activated by cyclic adenosine monophosphate (Epac) is a guanine nucleotide exchange factor and has been identified as a sensor of cyclic adenosine monophosphate (cAMP). Epac has been reported to mediate the cAMP signaling pathway in a number of pathological and physiological processes, such as cell inflammation, metabolism, and neurological dysfunction (6-8). After stress, Epac1 acts as a major response second messenger (9). In vitro, stimulation of excitant induces rapid translocation of cytosolic Epac1 to plasma membrane. Subsequently, Epac1 binds phosphatidic acid by its Disheveled/Egl-10/ pleckstrin domain and activates Rap signals, such as protein kinase $\mathrm{C}(\mathrm{PKC}), \mathrm{Akt}$, and mitogen-activated protein kinases (MAPKs) (10-12). In vivo, classical cAMP/protein kinase A (PKA), MAPKs and Akt-dependent pathways play an important role in hyperalgesic priming (13-15). Recently, an increase of Epac1 was reported in the dorsal root ganglion (DRG), which promoted the transition toward chronic pain. This might be a potential pathway for treating chronic pain (16-18). Then, we hoped to find both peripheral and central targets which were vital in the progress of chronic pain. Our previous experiments revealed that peripheral Epac1 was involved in hyperalgesia induced by SMIR. Specifically, this was associated with its effects on adhesive linkage disorder and vascular endothelial hyperpermeability (19). These disorders might contribute to the chronic, eutrophic, and inflammatory microenvironment in injuries. However, if spinal Epac1 plays an important role and the underlying mechanisms are essentially still unclear. Here, we firstly investigated the role of spinal Epac1 in the disruption of glial activity associated with CPSP. We present the following article in accordance with the ARRIVE reporting checklist (available at https://dx.doi.org/10.21037/atm-21-5384).

\section{Methods}

\section{Animals}

Male Sprague Dawley (SD) rats (200-250 g body weight, 8-10 weeks of age) were obtained from the Experimental Animal Center of Nantong University and maintained in a temperature-controlled room $\left(23 \pm 1{ }^{\circ} \mathrm{C}\right)$ with a 12 -h light/ dark cycle. Experiments were performed under the Guide for the Care and Use of Laboratory Animals. The protocols were approved by the Experimental Animal Protection and Care Committee of Nantong University (20170305-001). Water and food were freely available in the Laboratory of
Animal Behavior.

\section{Groups and drugs}

Rats were divided randomly into five groups and anesthetized with $10 \%$ chloral hydrate $(300 \mathrm{mg} / \mathrm{kg}$, i.p.). (I) The naive group received no treatment. (II) In the sham group, an incision was made on the skin of the medial thigh 3-4 mm medially to the saphenous vein. (III) In the SMIR group, the incision was bluntly separated 7 to $10 \mathrm{~mm}$ by exposing the superficial muscles, and the tip of the retractor was inserted into the superficial muscle to expand the incision to $2 \mathrm{~cm}$. The incision was sutured $1 \mathrm{~h}$ later with medical silk. (IV) In the SMIR + CE3F4 (Epac1 inhibitor) group, rats received intrathecal injection of Epac1, CE3F4 (Tocris, Bristol, UK) on day 7 after SMIR.CE3F4 was dissolved in dimethyl sulfoxide (DMSO) to achieve a $20 \mu \mathrm{g} / 20 \mu \mathrm{L}$ solution. (V) In the SMIR + vehicle group, rats received intrathecal injection of $10 \% \mathrm{DMSO}$ on day 7 after SMIR.

\section{Mechanical allodynia test}

Rats were adapted for $30 \mathrm{~min}$ on the metal screen in an organic glass box $\left(22 \times 12 \times 22 \mathrm{~cm}^{3}\right)$ before experiments The mechanical withdrawal threshold (MWT) was measured before surgery and on 1, 3, 7, 14, and $21 \mathrm{~d}$ after surgery; and 1, 3, 6, and $12 \mathrm{~h}$ after a single dose of drug. In the resting state, the electronic Von Frey filament cilia stimulator (North Coast Medical, Morgan Hill, CA, USA) was used to perform vertical stimulation to the hind paw. With Von Frey slightly bent as the total force standard, the duration was less than or equal to $4 \mathrm{~s}$. The test was repeated five times with a 5 -min interval. The appearance of paw withdrawal or paw licking was considered positive (at least three out of five applications), otherwise negative. The MWT was calculated according to the Chaplain up-down method (20).

\section{Western blotting}

After administration of deep anesthesia, the lumbosacral enlargement of the spinal cord was immediately removed and homogenized in ice-chilled tissue protein extraction reagent. The tissues were prepared as reported previously (19). The protein samples were transferred onto polyvinylidene fluoride membranes. The membranes were subsequently blocked with Tris-buffered saline solution containing 5.0\% 
skim milk powder for $2 \mathrm{~h}$ at room temperature and incubated with primary antibodies using Epac1 (1:300, Santa, Texas, USA) and glyceraldehyde 3-phosphate dehydrogenase (GAPDH) (1:5,000, Sigma, Burlington, MA, USA) at $4{ }^{\circ} \mathrm{C}$, overnight. After washing with Tris-buffered saline/Tween 20 , the membranes were incubated with the appropriate secondary antibody (1:5,000, Jackson, Pennsylvania, USA) for $2 \mathrm{~h}$ at room temperature. Immunoblots were detected using Tanon 2500 gel imaging system (Yph-Bio Co., Ltd., Beijing, China) with an enhanced ECL chemiluminescence detection kit (Absin, Shanghai, China). The results were quantified and analyzed using ImageJ (National Institutes of Health, Bethesda, MD, USA).

\section{Immunofluorescence staining}

We anesthetized the animals with $10 \%$ chloral hydrate (300 $\mathrm{mg} / \mathrm{kg}$, i.p.). Then, rats were transcardially perfused with saline, and the tissues were fixed using perfusion fixative ( $4 \%$ paraformaldehyde). The lumbosacral sections of the spinal cord were immediately removed and successively dehydrated in $20 \%$ and $30 \%$ sucrose solutions. The submerged tissues were frozen at $-20{ }^{\circ} \mathrm{C}$ and continuously sliced into $5 \mu \mathrm{m}$ sections. After washing with phosphate buffered saline (PBS)and blocking in 5\% serum for $2 \mathrm{~h}$ at room temperature, the sections were incubated with the respective primary antibodies Epac1 (1:50, Santa, Texas, USA), glial fibrillary acidic protein (GFAP) (1:1,000; Millipore, Burlington, MA, USA), Iba-1 (1:200, Wako, Osaka, Japan), and NeuN (1:500, Abcam, Cambridge, UK) (overnight, $4^{\circ} \mathrm{C}$ ). The tissue sections were then washed with PBS and separately incubated with the secondary antibodies for $2 \mathrm{~h}$ at room temperature. The secondary antibodies were $\mathrm{Cy} 3$-conjugated goat anti-rabbit immunoglobulin (IgG) (1:1,000, Jackson, Pennsylvania, USA) and fluorescein isothiocyanate (FITC)-conjugated goat anti-mouse IgG (1:1,000, Jackson, USA). Images were captured using a fluorescence microscope (Olympus, Shinjuku, Japan).

\section{Statistical analysis}

All data are expressed as means \pm SEM and were analyzed with SPSS Version 23 software. All statistical comparisons were performed with one-way or two-way analysis of variance (ANOVA) followed by the Bonferroni post hoc test. A value of $\mathrm{P}<0.05$ was considered as indicating statistical significance.

\section{Results}

\section{SMIR induced mechanical allodynia and the upregulation of Epac1 in the spinal cord}

Prior to surgery, there were no differences of MWT among the three groups. On day 1, both the sham and SMIR groups displayed a decline of MWT in the ipsilateral hindpaw $(\mathrm{P}<0.05)$. However, the MWT of the sham group returned to baseline from day 3 after surgery. The decline of MWT was observed on day 1 in the SMIR group and reached a stable period on day 7 after surgery. These changes lasted for at least 21 days during our observation period (Figure 1A). The data implied a successful establishment of the SMIR-induced CPSP model.

Then, we assessed the expression of Epac1 in the spinal cord using western blotting. The expression of Epac1 was gradually increased in the progression of the SMIRinduced CPSP model. Compared with the sham group, the expression of Epac1 significantly increased from day 3 after SIMR $(\mathrm{P}<0.05)$. This upregulation lasted for at least 21 days after SMIR (Figure 1B).

\section{In the later stage of hyperalgesia, Epac1 was expressed in spinal astrocytes after SMIR}

Hyperexcitability of glia and neurons after nerve injury has been strongly implicated in chronic pain (21). To elucidate the potential mechanism underlying CPSP in this model, we further examined the localization of Epac1 in the spinal cord on day 7 after SMIR (Figure 2). Notably, Epac1 immunoreactivity was colocalized primarily with astrocytes (GFAP) but not with microglial cells (ionized calcium binding adaptor molecule 1, Iba1) or neurons (neuronspecific nuclear protein, $\mathrm{NeuN}$ ).

\section{Inbibition of Epac1 alleviated mechanical allodynia induced by SMIR}

In the SMIR group, rats exhibited significant mechanical allodynia after surgery. On day 7 , intrathecal injection of Epac1 inhibitor CE3F4 significantly prevented and suppressed SMIR-induced mechanical allodynia $(\mathrm{P}<0.05)$. The upward tendency of MWT was in a time-dependent manner. In fact, the antinociceptive effect of $\mathrm{CE} 3 \mathrm{~F} 4$ started at $1 \mathrm{~h}$ after intrathecal injection and was sustained at least $6 \mathrm{~h}$ after injection $(\mathrm{P}<0.05)$. In addition, the antinociceptive effect of $\mathrm{CE} 3 \mathrm{~F} 4$ reached a peak at $3 \mathrm{~h}$ after injection and 
A

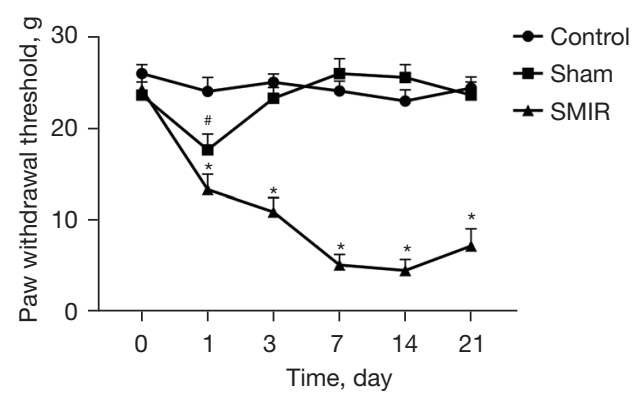

B

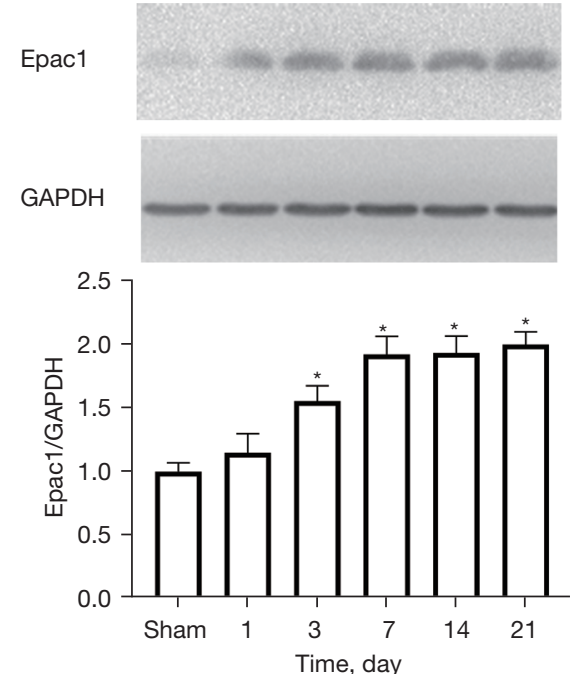

Figure 1 Development of mechanical allodynia and spinal expression of Epac1 in the skin/muscle incision and retraction (SMIR) group. (A) Changes of pain sensitivity in different groups were determined by mechanical withdrawal threshold (MWT) on the day before operation (baseline) and on 1, 3, 7, 14, and $21 \mathrm{~d}$ after operation $(\mathrm{n}=8)$. (B) Western blots and statistical analysis of spinal Epac1 at different times (n=5). *, $\mathrm{P}<0.05$ vs. sham group.
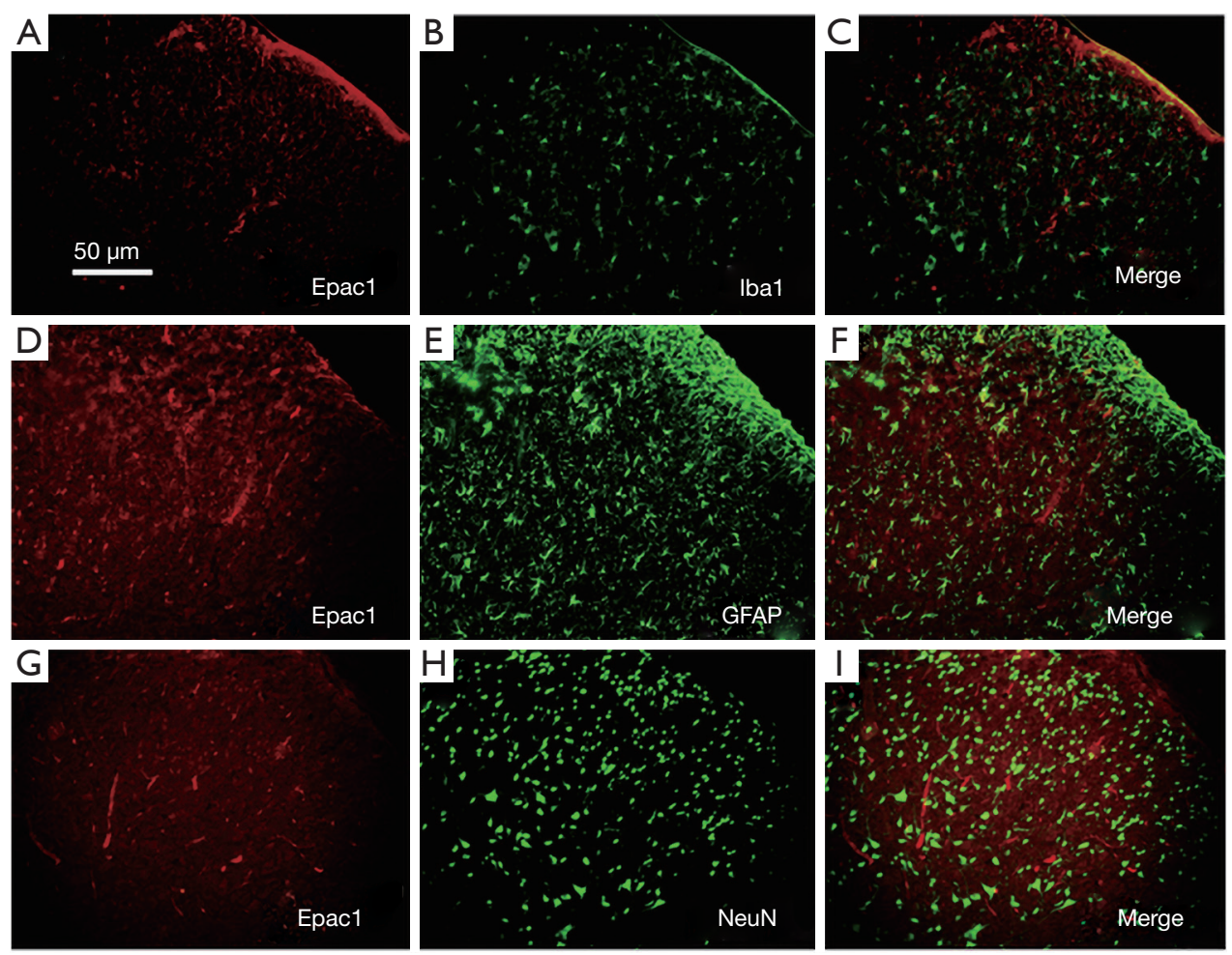

Figure 2 Immunofluorescence showed the colocalization of Epac1 (red, left line) microglial cells [ionized calcium binding adaptor molecule 1 (Iba1), green], astrocytes [glial fibrillary acidic protein (GFAP), green], and neurons [neuron-specific nuclear protein (NeuN), green] in the spinal cord $(\mathrm{n}=5)$. Tissues were collected on day 7 after skin/muscle incision and retraction. Magnification $\times 200$. Scale bar $=50 \mu \mathrm{m}$. 


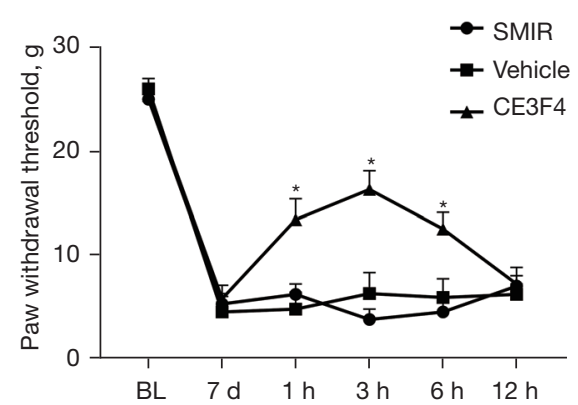

Figure 3 Intrathecal injection of Epac1 inhibitor CE3F4 alleviated mechanical allodynia induced by skin/muscle incision and retraction (SMIR) $(\mathrm{n}=8)$. Changes of pain sensitivity in different groups were determined by mechanical withdrawal threshold on the day before operation (baseline), $7 \mathrm{~d}$ after operation, and at 1, 3, 6 , and $12 \mathrm{~h}$ after different treatments. *, $\mathrm{P}<0.05$ vs. sham group.

disappeared at $12 \mathrm{~h}$ after injection (Figure 3). As controls, no effects were observed in rats with vehicle treatment $(\mathrm{P}>0.05)$.

\section{Inbibition of Epac1 suppressed the activation of astrocytes induced by SMIR}

Astrocytes are abundant in the central nervous system (CNS), and glia play an important role in different pain disorders (22-24). Therefore, we examined whether the hyperactivity of astrocytes during CPSP was regulated by Epac1. Consistent with earlier experiments, SMIR increased GFAP staining, indicating astrocytes activation in the dorsal horn of the spinal cordon day 7 (Figure 4). Intrathecal injection of Epac1 inhibitor CE3F4 significantly suppressed the expression of GFAP $3 \mathrm{~h}$ after treatment $(\mathrm{P}<0.05)$. However, no corresponding changes were observed in the vehicle treatment group $(\mathrm{P}>0.05)$.

\section{Discussion}

This experiment revealed a crucial role of Epac1 in the progress of CPSP. The present findings indicated that (I) SMIR induced long-lasting mechanical allodynia. (II) SMIR-induced postoperative hyperalgesia caused the upregulation of Epac1 and the activation of astrocytes in the spinal cord. (III) In the later stage of hyperalgesia, the upregulation of Epac1 was astrocytes-derived but not through microglial cells or neurons. (IV) Spinal blockade of Epac1 alleviated SMIR-induced postoperative hyperalgesia and hyperactivity of astrocytes. The findings might provide novel insights into the mechanism of CPSP.
Glial cell hyperactivity in reaction to the stimulation is involved in ischemia, trauma, and invading pathogens $(25,26)$. Gliosis and inflammation are important mediators of promoting a persistent pain state (27-29). Many studies have confirmed that hyperactivity of spinal microglia predominates in the early responses to peripheral nerve injury, and subsequently spinal astrocytes undergo activation and proliferation $(30,31)$. Then microglia and astrocytes release various mediators including proinflammatory cytokines, like TNF- $\alpha$, IL-1 $\beta$, etc., which ultimately contributes to hyperalgesia and allodynia (32-34). As expected, we found that the later-stage response to SMIR was dominated by the activation of astrocytes in the spinal cord. Cyclical AMP signaling has been confirmed as a primary pathway in the sensitization of nociceptors. Our previous experiments revealed that peripheral Epac1 was involved in hyperalgesia induced by SMIR. Specifically, this was associated with its effects on adhesive linkage disorder and vascular endothelial hyperpermeability (19). However, there are few reports about the relationship between Epac1 and spinal astrocytes in CPSP. In cultured astrocytes, $\beta$-adrenergic/Epac signaling regulates the morphological changes of astrocytes, which affects astrocyte and synaptic function (35). The expression of Epac is developmentally regulated in the damaged CNS of the rat. Additionally, Epac participates in DRG neurite outgrowth and neurite regeneration in the spinal cord (36). In our study, the upregulation of Epac1 was astrocyte-derived and not through microglia or neuron. Spinal inhibition of Epac1 suppressed the activation of astrocytes in the spinal cord. This might be a potential target for the treatment of CPSP. Reactive astrocytes are divided into toxic $\mathrm{A} 1$ astrocytes and neuroprotective A2 astrocytes. A1 astrocytes induce rapid death of neurons and oligodendrocytes, while A2 astrocytes promote neuronal survival and tissue repair $(37,38)$. Reactive astrogliosis can increase nutritional support and neuroprotection for the damaged neurons (39). Therefore, the role of A1 astrocytes in regulating neuroinflammation, energy metabolism, and synaptic plasticity during chronic pain and the mechanism of A2 astrocytes in alleviating the development of pain require further study.

In human prostate cancer cells, Epac1 acts as a proinflammatory modulator and upregulates MAPK and mechanistic target of rapamycin (mTOR) signaling pathways, which leads to the proliferation of cancer cells (40). Reports show that EPAC1 ${ }^{-1-}$ mice are protected against acute inflammatory pain and neuropathic pain $(41,42)$. The disturbance of phosphorylated Epac1, activated 

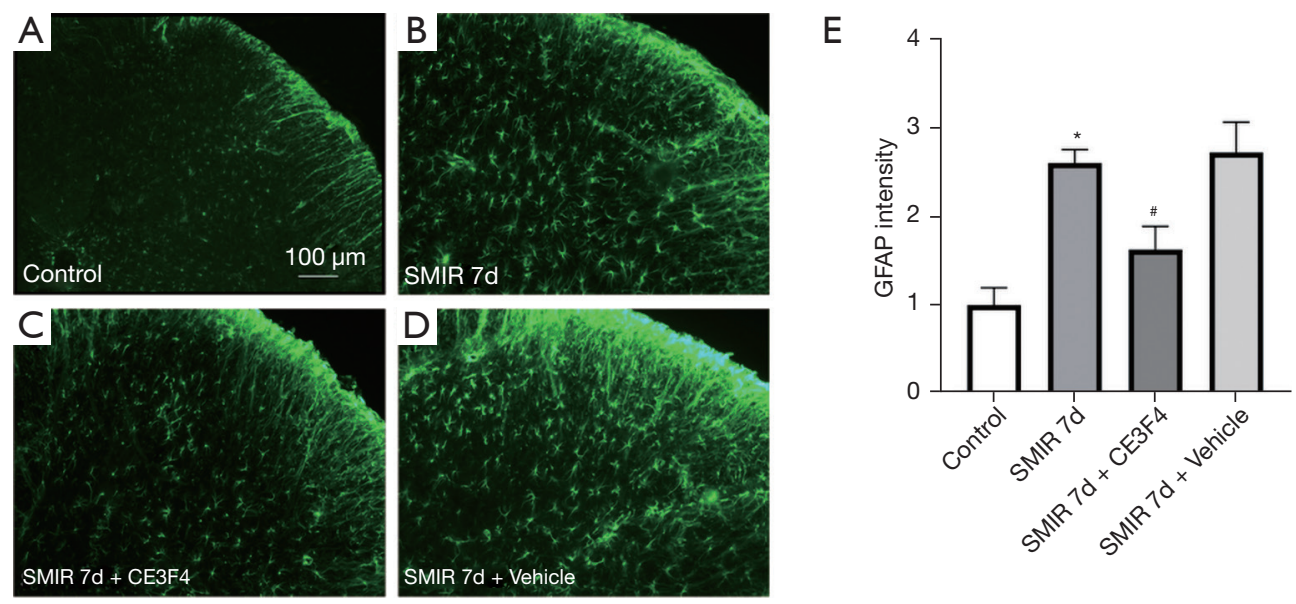

Figure 4 Intrathecal injection of Epac1 inhibitor CE3F4 inhibited skin/muscle incision and retraction of (SMIR)-induced hyperactivity of astrocytes $(\mathrm{n}=5)$. Immunostaining of astrocytes[glial fibrillary acidic protein (GFAP), green] in the ipsilateral dorsal horn before SMIR (A), $7 \mathrm{~d}$ after SMIR (B), $3 \mathrm{~h}$ after intrathecal injection of Epac1 inhibitor CE3F4 (C), and $3 \mathrm{~h}$ after intrathecal injection of vehicle (D). (E) The immunofluorescence densities of GFAP in different groups. *, $\mathrm{P}<0.05$ vs. control group; ${ }^{*}, \mathrm{P}<0.05$ vs. $7 \mathrm{~d}$ after SMIR.

by $\mathrm{G}$ protein-coupled receptor kinase 2 (GRK2) in dorsal root ganglion neurons, suppresses Ras-related protein 1 (Rap1) signaling and then promotes the transformation of acute inflammatory pain into chronic pain $(16,17)$. Epac1 is sufficient to maintain long-lasting latent sensitization of dorsal horn neurons in chronic inflammatory pain (43). During neuropathic pain, the Epac1-Piezo 2 axis also participates in the development of mechanical allodynia (41). Additionally, Epac1 is abundant in the vascular system (44). Epac1 can regulate mitochondrial fission and neointima formation, which contributes to the proliferation and phenotypic switch of vascular smooth muscle cells after vascular injury (45). We previously showed that Epac1 promotes the macrophage proliferation of macrophage and endothelial cells around the incision in SMIR (19). This might recruit a lot of proinflammatory cytokines and chemokines and remodel local nutrient-rich microenvironment around the incision. In combination, both peripheral and central Epac1 is vital in the progress of chronic pain. Also, the underlying mechanisms might be different in different positions or different models.

\section{Conclusions}

In conclusion, our study demonstrated an important role of spinal Epac1 in the development of CPSP. In the later stage of hyperalgesia, inhibition of spinal Epac1 signaling might elicit analgesic effects via the modulation of astroglial activity in the spinal cord.

\section{Acknowledgments}

Funding: This research was supported by the National Natural Science Foundation of China (81701106) and the Subsidized Projects of Nantong Science and Technology Bureau (JC2018055).

\section{Footnote}

Reporting checklist: The authors have completed the ARRIVE reporting checklist. Available at https://dx.doi. org/10.21037/atm-21-5384

Data Sharing Statement: Available at https://dx.doi. org/10.21037/atm-21-5384

Conflicts of Interest: All authors have completed the ICMJE uniform disclosure form (available at https://dx.doi. org/10.21037/atm-21-5384). All authors report that this research was supported by the National Natural Science Foundation of China (81701106) and the Subsidized Projects of Nantong Science and Technology Bureau (JC2018055). The authors have no other conflicts of interest to declare. 
Ethical Statement: The authors are accountable for all aspects of the work in ensuring that questions related to the accuracy or integrity of any part of the work are appropriately investigated and resolved. The protocols were approved by the Ethics Committee of Affiliated Hospital of Nantong University (20170305-001). Experiments were performed under the Guide for the Care and Use of Laboratory Animals.

Open Access Statement: This is an Open Access article distributed in accordance with the Creative Commons Attribution-NonCommercial-NoDerivs 4.0 International License (CC BY-NC-ND 4.0), which permits the noncommercial replication and distribution of the article with the strict proviso that no changes or edits are made and the original work is properly cited (including links to both the formal publication through the relevant DOI and the license). See: https://creativecommons.org/licenses/by-nc-nd/4.0/.

\section{References}

1. Ching YY, Wang C, Tay T, et al. Altered Sensory Insular Connectivity in Chronic Postsurgical Pain Patients. Front Hum Neurosci 2018;12:483.

2. Kim DH, Pearson-Chauhan KM, McCarthy RJ, et al. Predictive Factors for Developing Chronic Pain After Total Knee Arthroplasty. J Arthroplasty 2018;33:3372-8.

3. Fregoso G, Wang A, Tseng K, et al. Transition from Acute to Chronic Pain: Evaluating Risk for Chronic Postsurgical Pain. Pain Physician 2019;22:479-88.

4. Zeilhofer HU, Benke D, Yevenes GE. Chronic pain states: pharmacological strategies to restore diminished inhibitory spinal pain control. Annu Rev Pharmacol Toxicol 2012;52:111-33.

5. Chapman CR, Vierck CJ. The Transition of Acute Postoperative Pain to Chronic Pain: An Integrative Overview of Research on Mechanisms. J Pain 2017;18:359. e1-359.e38.

6. Parnell E, Yarwood SJ. Interactions between Epac1 and ezrin in the control of endothelial barrier function. Biochem Soc Trans 2014;42:274-8.

7. Baameur F, Singhmar P, Zhou Y, et al. Epac1 interacts with importin $\beta 1$ and controls neurite outgrowth independently of cAMP and Rap1. Sci Rep 2016;6:36370.

8. Bouvet M, Blondeau JP, Lezoualc'h F. The Epac1 Protein: Pharmacological Modulators, Cardiac Signalosome and Pathophysiology. Cells 2019;8:1543.

9. de Rooij J, Rehmann H, van Triest M, et al. Mechanism of regulation of the Epac family of cAMP-dependent RapGEFs. J Biol Chem 2000;275:20829-36.

10. Ponsioen B, Gloerich M, Ritsma L, et al. Direct spatial control of Epac1 by cyclic AMP. Mol Cell Biol 2009;29:2521-31.

11. Gao M, Ma Y, Bast RC Jr, et al. Epac1 knockdown inhibits the proliferation of ovarian cancer cells by inactivating AKT/Cyclin D1/CDK4 pathway in vitro and in vivo. Med Oncol 2016;33:73.

12. Mei FC, Qiao J, Tsygankova OM, et al. Differential signaling of cyclic AMP: opposing effects of exchange protein directly activated by cyclic AMP and cAMPdependent protein kinase on protein kinase $\mathrm{B}$ activation. J Biol Chem 2002;277:11497-504.

13. Asiedu MN, Tillu DV, Melemedjian OK, et al. Spinal protein kinase $M \zeta$ underlies the maintenance mechanism of persistent nociceptive sensitization. J Neurosci 2011;31:6646-53.

14. Zhang T, Zhang N, Zhang R, et al. Preemptive intrathecal administration of endomorphins relieves inflammatory pain in male mice via inhibition of p38 MAPK signaling and regulation of inflammatory cytokines. J Neuroinflammation 2018;15:320.

15. Hu B, Xu G, Zhang X, et al. Paeoniflorin Attenuates Inflammatory Pain by Inhibiting Microglial Activation and Akt-NF- $\kappa B$ Signaling in the Central Nervous System. Cell Physiol Biochem 2018;47:842-50.

16. Singhmar P, Huo X, Eijkelkamp N, et al. Critical role for Epac1 in inflammatory pain controlled by GRK2mediated phosphorylation of Epac1. Proc Natl Acad Sci U S A 2016;113:3036-41.

17. Wang H, Heijnen CJ, van Velthoven CT, et al. Balancing GRK2 and EPAC1 levels prevents and relieves chronic pain. J Clin Invest 2013;123:5023-34.

18. Gu Y, Li G, Chen Y, et al. Epac-protein kinase C alpha signaling in purinergic P2X3R-mediated hyperalgesia after inflammation. Pain 2016;157:1541-50.

19. Pan P, Huang SS, Shen SR, et al. Role of p120 Catenin in Epac1-Induced Chronic Postsurgical Pain in Rats. Pain Res Manag 2019;2019:9017931.

20. Dixon WJ. Staircase bioassay: the up-and-down method. Neurosci Biobehav Rev 1991;15:47-50.

21. Basbaum AI, Bautista DM, Scherrer G, et al. Cellular and molecular mechanisms of pain. Cell 2009;139:267-84.

22. Ying YL, Wei XH, Xu XB, et al. Over-expression of $\mathrm{P} 2 \mathrm{X} 7$ receptors in spinal glial cells contributes to the development of chronic postsurgical pain induced by skin/ muscle incision and retraction (SMIR) in rats. Exp Neurol 
2014;261:836-43.

23. Jiang BC, Cao DL, Zhang X, et al. CXCL13 drives spinal astrocyte activation and neuropathic pain via CXCR5.J Clin Invest 2016;126:745-61.

24. Ji RR, Donnelly CR, Nedergaard M. Astrocytes in chronic pain and itch. Nat Rev Neurosci 2019;20:667-85.

25. Austin PJ, Moalem-Taylor G. The neuro-immune balance in neuropathic pain: involvement of inflammatory immune cells, immune-like glial cells and cytokines. J Neuroimmunol 2010;229:26-50.

26. Liou JT, Liu FC, Mao CC, et al. Inflammation confers dual effects on nociceptive processing in chronic neuropathic pain model. Anesthesiology 2011;114:660-72.

27. Gosselin RD, Suter MR, Ji RR, et al. Glial cells and chronic pain. Neuroscientist 2010;16:519-31.

28. Nakagawa T, Kaneko S. Spinal astrocytes as therapeutic targets for pathological pain. J Pharmacol Sci 2010;114:347-53.

29. Schomberg D, Olson JK. Immune responses of microglia in the spinal cord: contribution to pain states. Exp Neurol 2012;234:262-70.

30. Romero-Sandoval A, Chai N, Nutile-McMenemy N, et al. A comparison of spinal Iba1 and GFAP expression in rodent models of acute and chronic pain. Brain Res 2008;1219:116-26.

31. Tanga FY, Raghavendra V, DeLeo JA. Quantitative real-time RT-PCR assessment of spinal microglial and astrocytic activation markers in a rat model of neuropathic pain. Neurochem Int 2004;45:397-407.

32. Hald A, Nedergaard S, Hansen RR, et al. Differential activation of spinal cord glial cells in murine models of neuropathic and cancer pain. Eur J Pain 2009;13:138-45.

33. Ren BX, Gu XP, Zheng YG, et al. Intrathecal injection of metabotropic glutamate receptor subtype 3 and 5 agonist/antagonist attenuates bone cancer pain by inhibition of spinal astrocyte activation in a mouse model. Anesthesiology 2012;116:122-32.

34. Wang LN, Yang JP, Zhan Y, et al. Minocycline-induced reduction of brain-derived neurotrophic factor expression in relation to cancer-induced bone pain in rats. J Neurosci Res 2012;90:672-81.

35. Vardjan N, Kreft M, Zorec R. Dynamics of $\beta$-adrenergic/ cAMP signaling and morphological changes in cultured astrocytes. Glia 2014;62:566-79.

36. Murray AJ, Shewan DA. Epac mediates cyclic AMPdependent axon growth, guidance and regeneration. Mol Cell Neurosci 2008;38:578-88.

37. Liddelow SA, Guttenplan KA, Clarke LE, et al. Neurotoxic reactive astrocytes are induced by activated microglia. Nature 2017;541:481-7.

38. Miller SJ. Astrocyte Heterogeneity in the Adult Central Nervous System. Front Cell Neurosci 2018;12:401.

39. Pekny M, Wilhelmsson U, Pekna $M$. The dual role of astrocyte activation and reactive gliosis. Neurosci Lett 2014;565:30-8.

40. Misra UK, Pizzo SV. Evidence for a pro-proliferative feedback loop in prostate cancer: the role of Epac1 and COX-2-dependent pathways. PLoS One 2013;8:e63150.

41. Eijkelkamp N, Linley JE, Torres JM, et al. A role for Piezo2 in EPAC1-dependent mechanical allodynia. Nat Commun 2013;4:1682.

42. Singhmar P, Huo X, Li Y, et al. Orally active Epac inhibitor reverses mechanical allodynia and loss of intraepidermal nerve fibers in a mouse model of chemotherapy-induced peripheral neuropathy. Pain 2018;159:884-93.

43. Fu W, Nelson TS, Santos DF, et al. An NPY Y1 receptor antagonist unmasks latent sensitization and reveals the contribution of protein kinase A and Epac to chronic inflammatory pain. Pain 2019;160:1754-65.

44. Laurent AC, Bisserier M, Lucas A, et al. Exchange protein directly activated by cAMP 1 promotes autophagy during cardiomyocyte hypertrophy. Cardiovasc Res 2015;105:55-64.

45. Wang H, Robichaux WG, Wang Z, et al. Inhibition of Epac1 suppresses mitochondrial fission and reduces neointima formation induced by vascular injury. Sci Rep 2016;6:36552.
Cite this article as: She Q, Chen J, Zhong C, Huang S, Lu C, Qin Y. Exchange proteins directly activated by cyclic adenosine monophosphate inhibitor reverses mechanical allodynia via the modification of astrocytes activity in the spinal cord. Ann Transl Med 2021;9(22):1656. doi: 10.21037/atm-21-5384 